\title{
Investigation on natural frequency of an optimized elliptical container using real-coded genetic algorithm
}

\section{Abstract}

This study introduces a method based on real-coded genetic algorithm to design an elliptical shaped fuel tank. This method enhances the advantage of the system such as roll stability, and reduces disadvantages like fluid c.g. height and overturning moment. These parameters corresponding to the elliptical tanks with different filling levels are properly optimized. Moreover the effects of these optimized shapes on natural sloshing frequency are investigated. Comparing presented results with experimental ones indicate the reliability and accuracy of the present work. In addition, a new method based on genetic algorithm, which enhances tank rollover threshold, is presented. This optimization enhances roll stability, although reducing the natural sloshing frequency in comparison to cylindrical tanks. In contrast, the sloshing frequency of the optimized elliptical tank is enhanced in compare with conventional elliptical tanks, which is considered as an advantageof the presented work.

\section{Keywords}

Genetic algorithm, sloshing, optimization, tank vehicle, overtur-

ning moment.

\author{
M. H. Shojaeefard ${ }^{a}$ \\ R. Talebitooti ${ }^{a}$ \\ S. Yarmohammadi Satri ${ }^{a, *}$ \\ Mohammad Hassan Amiryoon ${ }^{b}$ \\ School of Mechanical Engineering., Iran \\ University of Science and Technology, Teh- \\ ran, Iran. \\ b Center of Excellence for Power System \\ Automation and Operation, Department of \\ Electrical Engineering, Iran University of \\ Science and Technology, Tehran, Iran \\ Received in 29 Dec 2012 \\ In revised form 09 Apr 2013 \\ *Author email: yysadegh@yahoo.com
}

\section{INTRODUCTION}

Generally, Rollover accidents are one of the most common types of accident that occur in commercial vehicles carrying fluid. Since most of the time, these tankers carry dangerous liquids such as ammonia, gasoline, and fuel oils, therefore stability of partially filled liquid cargo vehicles are of great importance (Acarman, 2006). Sloshing is a fluid oscillation phenomenon caused by the tank motion. Fluid oscillation depends on the tank geometry, filling conditions, and frequency range inside the tanker (Budiansky, 1960). Besides, sloshing frequency and magnitude of sloshing forces are also dependent on these parameters (Hasheminejad and Aghabeig, 2011). If the sloshing frequency is close enough to the structural natural frequency, resonance will occur (Ibrahim, 1975). In addition, the stability is one of the most important points in design of vehicles used for carrying and storing objects and substances. There are many researches devoting on sloshing fre- 
quency of tankers. Nonlinear dynamic response of a system which is subjected to harmonic external force, have been studied by interacting lowest fluid sloshing mode with the various modes of structure. Internal resonance and combined resonance profile are presented as results (Ibrahim, 1988). Nakagawa and Ikeda (1997) studied theoretically and experimentally non linear vibrations of a two-dimensional rectangular structure and water sloshing. Aliabadi et al. (2003)conducted a comparison between the numerical analysis, fluid mechanics, and mechanical models. NavierStokes equations have been solved with the aid of finite element method to measure accuracy of pendulum model under constant lateral acceleration. Based on these results, both methods validate each other under low fuel tank filling. The difference between ranges of frequencies in these two methods is considerable when there is a large amount of fuel in the tank. Mechanical models such as roll plane model have been developed as an alternative approach to the study of fluid sloshing. The roll plane model is determined by center of gravity, resulting force and torque, frequency of oscillation, and mass and inertia characteristics of the mechanical system, which is equal to tank fluid slosh ( $\mathrm{Li}$ and Wang, 2012). The roll stability of a partially filled vehicle is influenced by both the c.g. height and the magnitude of lateral load transfer. Different crosssections have been analyzed to optimize the roll stability. The circular cross section has a high center of fluid location, but considerably less lateral load transfer with low fill volumes under a steady turning lateral acceleration field. On the other hand, modified oval tanks when compared to circular tanks yield lower c.g. height and relatively larger lateral load transfer with low fill volumes. Therefore, the modified oval tanks represent higher roll stability limits than circular tanks (Kang et al., 1999; Rakheja et al., 1988; Rakheja, 2002; Hyun-Soo, 2008). In order to optimize the elliptical shape of road containers, a numerical analysis is developed which minimizes overturning moment according to the position of fluid c.g. (Popov, 1996). The results indicate that the optimal ratio of height and width in elliptical tankers has decreased in terms of lateral momentum.Mostresearches in the field ofsustainabletanker concentrate on enhancing roll stability with the usage of baffles. Optimizing cross-section and effects of natural frequency on optimized tanks have not been considered in recent studies.

In this article, the elliptical tank shape is initially optimized based on real value method of genetic algorithm. Then, overturning moment and fluid c.g. height are minimized simultaneously. The obtained cross-sections enhance roll stability in different filling levels. The influence of GA parameters such as mutation rate and population on the accuracy and rapidity of results convergence are properly described. Moreover, the natural frequency of optimized shape, and also the effects of optimization on sloshing frequency are discussed. Finally the results of the presented research are compared with those of conventional circular, elliptical, and generic tank crosssections.

\section{QUASI-STATIC EQUILIBRIUM FORMULATION}

Assuming the quasi-static fluid sloshing, the roll plane model of a tanker is established. Lateralaccelerationandroll of the tankercausesmoving the fluidmasscenterfrom pointto point $c_{l}$ to the point $x_{l}$. Inthis study, therotationangleisassumed to be small andviscousfluid is Negligible. Eq. (1)shows theshape ofanellipticaltanker.

Latin American Journal of Solids and Structures 11(2014) 113 - 129 


$$
\frac{x^{2}}{a^{2}}+\frac{(y-b)^{2}}{b^{2}}=1
$$

where $x$ and $y$ are the coordinates of elliptical geometry, $2 a$ and $2 b$ are elliptical height and width respectively, Eq. (1) can be rewritten as:

$$
y=b\left(1 \pm \sqrt{1-\left(\frac{x}{a}\right)^{2}}\right)
$$

To obtain themass andcenter of gravity,equationswhichrepresentsthe fluidmotion,should beusedrepeatedly. The equation offluid free surface withoutsprung mass roll angleand lateral acceleration represented as:

$$
y=-h_{0}^{(i)}, \quad i=1, \ldots, n
$$

while Equations (1-3) are solved simultaneously the intersecting points of the fluid free surface containing $\left(x_{1}, y_{1}\right)$ and $\left(x_{2}, y_{2}\right)$ are obtained (Figure 1). After comparing $h_{0}{ }^{(i)}$ with themaximum and minimumvalues ofthe arcs limits,cross-sectional areaare obtained.

Since $\left(x_{1}, y_{1}\right)$ and $\left(x_{2}, y_{2}\right)$ are symmetricalpoints with respect toyaxis in theabsence oflateral acceleration and roll angle of sprung mass, therefore $x_{1}=-x_{2}$ and volumeoffluidper unit of tank lengthis predictedasfollows:

$$
A_{0}^{(i)}=2 \int_{0}^{y_{p}} \int_{f_{1}(x)}^{h_{0}(i)} d x d y
$$

$f_{1}(x)$ expresses the equationoftank geometry. The equation offree fluid surface under steady turning presented as:

$$
y=-\left(\frac{\varphi_{S}-a_{y}}{1+\varphi_{S} a_{y}}\right) x+\alpha^{(i)} \quad, i=1, \ldots, n
$$

$\boldsymbol{\varphi}_{s}$ is the roll angle of sprung mass, $a_{y}$ is the lateral acceleration in $g$ (gravity acceleration) unit and $\alpha^{(i)}$ is intersection of the $y$-axis withthe free surface ofthe fluid. 


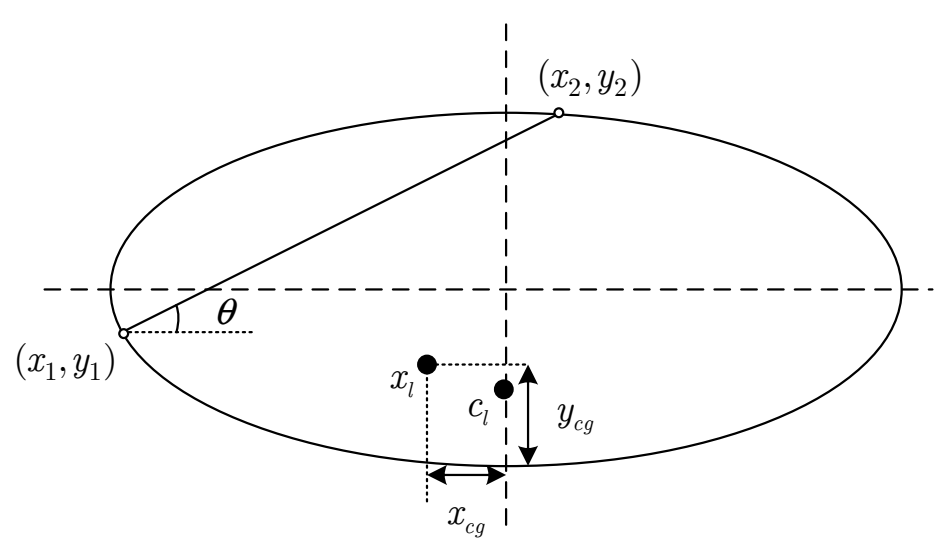

Figure 1 Elliptical parameters under steady lateral acceleration

The totalvolume offluidper unit lengthis compared with theinitial volumeinthe case that $a_{y}=0$ and $\varphi_{s}=0$. The error values corresponding to fluid volume are estimated as:

$$
\varepsilon=\left|A^{(i)}-A_{0}^{(i)}\right|
$$

Theprocess would be repeated again until the error function rate goes beyond the allowable error.

$$
\left\{\begin{array}{l}
\bar{Y}_{l}^{(i)}=\frac{1}{A^{(i)}} \int_{y_{p}}^{y_{q}} \int_{f_{1}(i)}^{f_{2}^{(i)}} y d y d x ; i=1,2, \ldots, 10 \\
\bar{X}_{l}^{(i)}=\frac{1}{A^{(i)}} \int_{y_{p}}^{y_{q}} \int_{f_{1}(i)}^{f_{2}^{(i)}} x d y d x ; i=1,2, \ldots, 10
\end{array}\right.
$$

For one iteration if $b \leq h \leq 2 b$, cross-sectional area $\left(A_{h}\right)$ and fluid c.g. height $\left(y_{c g}\right)$ are represented as:

$$
\begin{gathered}
A_{h}=\pi a b-\alpha \\
\alpha=2\left\{x_{h} b-x_{h} h+\frac{b}{2 a}\left[x_{h} \sqrt{a^{2}-x_{h}^{2}}+a^{2} \sin ^{-1}\left(\frac{x_{h}}{a}\right)\right]\right\} \\
y_{C G}=\frac{\pi a b^{2}-\alpha \beta}{A_{h}}
\end{gathered}
$$

while

$$
\beta=2 b-y_{C G}
$$




\section{TANK VEHICLE ROLL PLANE MODEL}

In order to optimize the tank cross-section, c.g. height and overturning moment are in challenge to each other. In low filling, circular cross-section presents more stability, as its overturning moment is less than elliptical tankers. The fluid c.g. height is dominated in high filling volumes. Therefore an elliptical tanker which has a lower center of gravity, represents more stability. In addition lateral and vertical liquid load shifts affect vehicle roll stability. In other handliquid load shift reduces as vehicle roll stability increases. This happens by minimizing overturning moment due to lateral acceleration and fluid c.g. height. The overturning moment is calculated with the geometric variables such as elliptical height and width (that is employed for computing fluid weight $(W)$, vertical and horizontal component of fluid c.g. $\left(X_{c g}, Y_{c g}\right)$ ) (Romero, 2005; Romero, 2007). In order to perform the optimization process, a vehicle model based on the one presented in Figure 2 with characteristics listed in Table 1 is employed. The fluid overturning moment is obtained by solving dynamical equations which are shown in the following.

$$
\begin{gathered}
\left\{\begin{array}{l}
m_{s} \ddot{z}_{s}=F_{s, k, o}+F_{s, c, o}+F_{s, c, i}+F_{s, k, i}-m_{s} g \\
I_{s} \ddot{\theta}_{s}=\left(F_{s, k, o}-F_{s, k, i}\right) s+\left(F_{s, c, o}-F_{s, c, i}\right) s+m_{s}\left(g-y_{s}\right)\left[\theta_{s}\left(h_{c s}-h_{r s}\right)\right]+M_{z}
\end{array}\right. \\
\left\{\begin{array}{c}
m_{u} \ddot{y}_{u}=F_{t, k, o}+F_{t, c, o}+F_{t, c, i}+F_{t, k, i}-\left(F_{s, k, o}+F_{s, c, o}+F_{s, c, i}+F_{s, k, i}\right)-m_{u} g \\
I_{u} \ddot{\theta}_{u}=\left(F_{t, k, o}-F_{t, k, i}\right) b+\left(F_{t, c, o}-F_{t, c, i}\right) b-\left(F_{s, k, o}-F_{s, k, i}\right) s-\left(F_{s, c, o}-F_{s, c, i}\right) s \\
+m_{u}\left(g-y_{u}\right)\left[\theta_{u}\left(h_{c a}-h_{r a}\right)\right]+m_{u}\left(a_{L}-y_{L}\right)\left(h_{c u}-h_{r u}\right)
\end{array}\right.
\end{gathered}
$$

For the sprung mass following equations can be written as:

$$
\begin{aligned}
& F_{s, k, o}=k_{s}\left(y_{s}+s \theta_{s}\right) \\
& F_{s, c, o}=c_{s}\left(\dot{y}_{s}+s \dot{\theta}_{s}\right) \\
& F_{s, k, i}=k_{s}\left(y_{s}-s \theta_{s}\right) \\
& F_{s, c, i}=c_{s}\left(\dot{y}_{s}-s \dot{\theta}_{s}\right)
\end{aligned}
$$

For the unsprung mass following equations can be written as:

$$
\begin{gathered}
F_{t, k, o}=k_{t}\left(y_{u}+b \theta_{u}\right) \\
F_{t, c, o}=c_{t}\left(\dot{y}_{u}+b \dot{\theta}_{u}\right) \\
F_{t, k, i}=k_{t}\left(y_{u}-b \theta_{u}\right) \\
F_{t, c, i}=c_{t}\left(\dot{y}_{u}-b \dot{\theta}_{u}\right) \\
M_{0}=W\left[\left(Y_{c g} \cos \varphi-X_{c g} \sin \varphi\right) a+Y_{c g} \sin \varphi+X_{c g} \cos \varphi\right]
\end{gathered}
$$




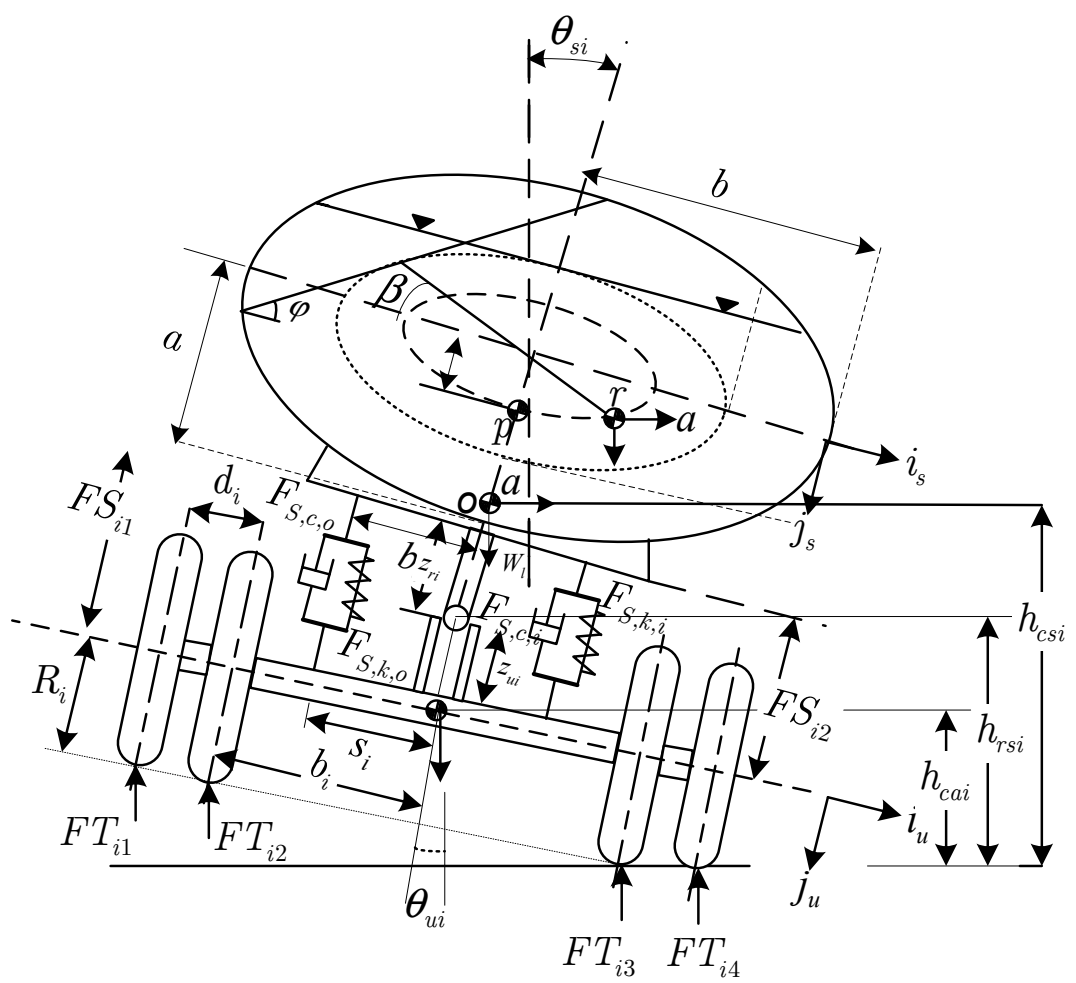

Figure 2 Roll plane model of elliptical tankers

Table 1 Specification of tanker used in roll plane model

\begin{tabular}{cc}
\hline \hline Tank truck characteristics & \\
\hline$m_{s}$, sprung mass & $5000 \mathrm{~kg}$ \\
$m_{u}$, unsprung mass & $1000 \mathrm{~kg}$ \\
$m_{c}$, cargo mass & $1000 \mathrm{~kg}$ \\
$k_{s}$, suspension stiffness & $1000 \mathrm{kN} / \mathrm{m}$ \\
$c_{s}$, suspension damping & $15 \mathrm{kNs} / \mathrm{m}$ \\
$k_{t}$, dual tire stiffness & $1400 \mathrm{kN} / \mathrm{m}$ \\
$c_{t}$ dual tire damping & $2.5 \mathrm{kNs} / \mathrm{m}$ \\
b, half track width & $1.0 \mathrm{~m}$ \\
s, half spring spacing & $0.7 \mathrm{~m}$ \\
$h_{c s}$ sprung mass c.g. height & $1.5 \mathrm{~m}$ \\
$h_{c a}$, axle c.g. height & $0.6 \mathrm{~m}$ \\
$h_{r s}$, sprung mass roll center height & $1.0 \mathrm{~m}$ \\
R, turning radius & $250 \mathrm{~m}$ \\
\hline
\end{tabular}

where $m_{s}, m_{u}$ and $m_{c}$ are sprung mass, un-sprung mass and cargo mass. $k_{s}$ is suspension stiffness, $c_{s}$ suspension damping, $k_{t}$ dual tire stiffness, $c_{t}$ dual tire damping, $b$ half track width, $s$ half spring spacing, $h_{c s}$ c.g. height of sprung mass, $h_{r s}$ height of sprung mass roll center. $F_{s, k, o}$ and $F_{s, k, i}$ are inner and outer spring forces in sprung mass. $F_{s, c, o}$ and $F_{s, c, i}$ are inner and outer damping forces in 
unsprung mass. $F_{t, k, o}$ and $F_{t, k, i}$ are inner and outer spring forces in unsprung mass. $F_{t, c, o}$ and $F_{t, c, i}$ are inner and outer damping forces in unsprung mass. $\theta_{s}$ and $\theta_{u}$ are roll angle of sprung mass and unsprung mass. The gradient of the liquid free surface is computed as Eq (17).

$$
\varphi=\frac{A+\theta_{s}}{1+A \theta_{s}}
$$

In order to verify the presented roll plane model, an elliptical tank with the tank width of 1.6 $m$, height of $0.8 \mathrm{~m}$, tank compartment length of $2.5 \mathrm{~m}$ and cross sectional area of $4 \mathrm{~m}^{2}$ is considered. These characteristics are applied based on dimensions of Popov (1996) tank. The results of overturning moment for this particular shape are compared with those represented by Popov (1996). As depicted in the Figure 3 the excellent agreements are achieved which shows the reliability of the model.

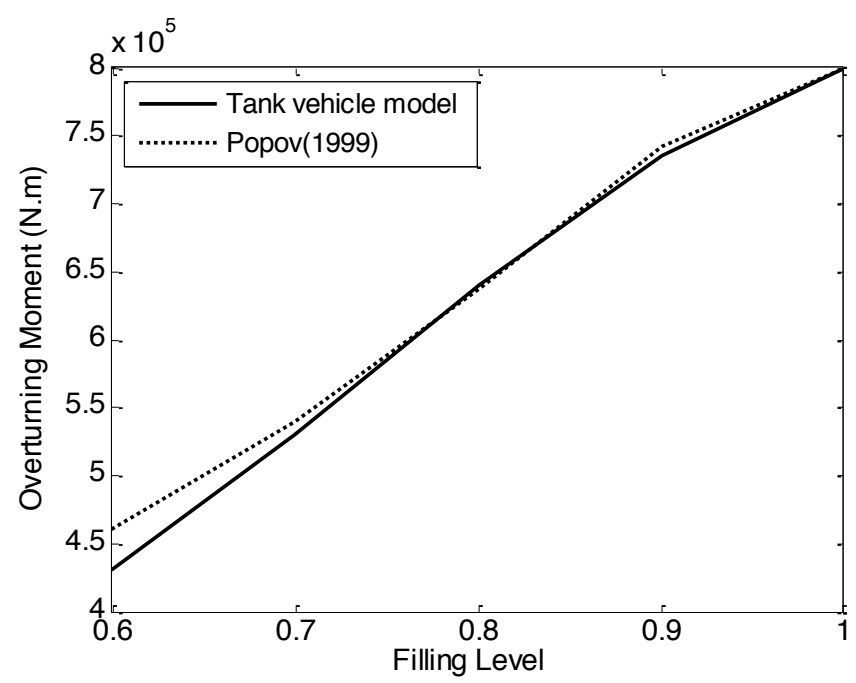

Figure 3 Validation of tank vehicle roll plane model based on overturning moment

\section{NATURAL SLOSHING FREQUENCY}

Fluid natural frequency should not be located near the tank resonance range in tank maneuvers. Natural sloshing frequency increases with enhancement of roll stability and fluid c.g. height in elliptical and circular tankers (Romero, 2007).Romero (2005) used two experimental and theoretical methods to estimate sloshing frequencies. The experimental method is based on effects of tank filling level and tank shape on the sloshing frequency under c.g. fluid shift. In general, longitudinal sloshing frequency is greater than the lateral sloshing frequency. This means that lateral natural frequency is more important than the longitudinal. In other word, the resonance phenomena occurs in lateral mode. In the theoretical method, the wave velocity equation is obtained using some simplifications including infinite fluid depth, and fixed and variable flow. In an ideal incompressible liquid, fluid surface velocity in rotating mode is a function of wave length $\lambda$, tank depth 
$h$, and gravity acceleration $g$. It is independent of other features such as fluid density. In general, the natural sloshing frequency in a rectangular tank is expressed as follows:

$$
c=\lambda f=\left(\frac{g}{k} \tanh k h\right)^{\frac{1}{2}}
$$

The resonance frequency in slow variation of speed is presented as:

$$
f=\frac{\bar{c}}{2 L}
$$

where $L$ is the wave length and $\bar{c}$ indicates the average wave speed and it is computed by the Eq. (19), as follows:

$$
\bar{c}=\frac{\frac{L}{2}}{\int_{0}^{\frac{L}{2}} \frac{d x}{c(x)}}
$$

The effect of wave length or in other word tank width especially for elliptical tank, in the case of fast wave speed variation should be mentioned in Eq. (21). The wave speed diminishes as a result of rapid depth difference, in both ends of elliptical surface. Therefore effective wave length $\left(L_{c}\right)$ is actually shorter than the value measured in shape $(L)$. Eq. (18) is used for determining sloshing frequency when the wave speed variation is in a limited band as Eq. (20).

$$
\begin{gathered}
\left|\frac{d \breve{c}(x)}{d x}\right| \geq a_{c r i t} \\
\left|\frac{d \breve{c}\left( \pm \frac{L_{c}}{2}\right)}{d x}\right| \geq a_{c r i t}
\end{gathered}
$$

Comparing the results of both experimental and theoretical methods indicates the deviation of $10 \%$ approximation. Natural sloshing frequencies for different shaped containers are obtained through substituting Eq. (23) $\left(h_{e q}\right)$ with $h$ in Eq. (18) which is shown in Figure 4 The equivalent fluid depth $h_{e q}$ is determined as a function of the liquid cross-sectional area and the fetch length $L$.

$$
h_{e q}=\frac{A_{\text {area }}}{L}
$$




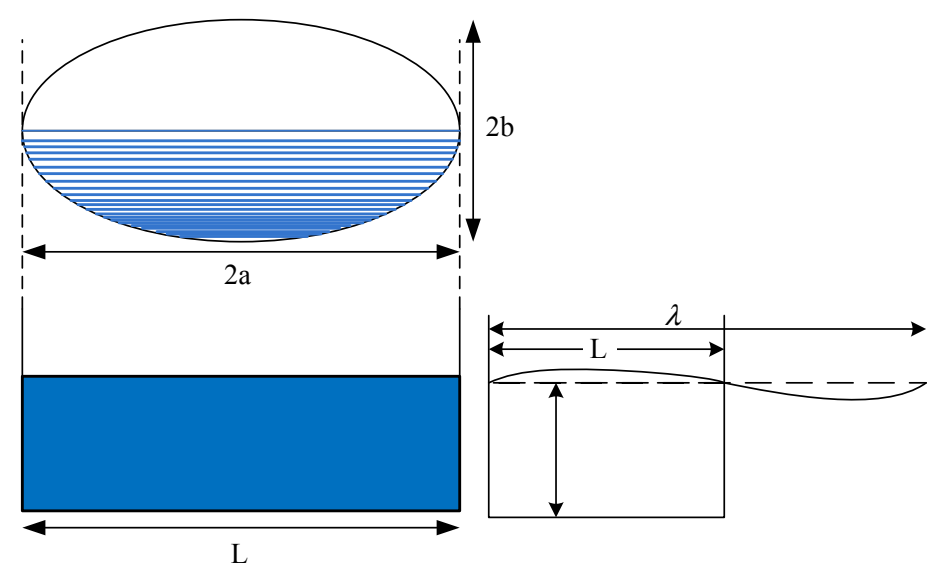

Figure 4 Equivalent fluid depth for a non-rectangular container

\section{GENETIC ALGORITHM}

The evolution algorithmspresent optimization techniques that are used in a wide range of operations such as parametric optimization, research, automatic generation of computer programs, and combined issues (Salehi and Tavakkoli-Moghaddam, 2009; Corriveau et al., 2001). Contrary to the traditional optimization techniques which use derivation in optimization evolutionary algorithms and utilize fitness function, evolutionary algorithms represent a set of individuals on optimization. The search region is divided into subsets that make it possible to achieve the general optimal point. The natural evolution operates by means of three main processes of evolution, mutation and selection. The main object of the mutation is producing new population and maintaining variation in the natural population. Effects of mutation rate selection on quickness and accuracy of fitness function convergence are studied in this paper. The crossover operator is used for transmitting good properties of the two populations as parents to a new population called offspring (Figure 5).

$$
\begin{aligned}
& \begin{array}{|l|l|l|l|l|l|l|l|l|l|l|l|}
\hline 2 & 1.5 & 3.1 & 4.2 & 5.1 & 6.3 & 4.6 & 3.1 & 2.5 & 1.2 & 3.4 & 5.3 \\
\hline
\end{array} \\
& \begin{array}{|l|l|l|l|l|l|l|l|l|l|l|l|}
\hline 3.2 & 4.1 & 2.4 & 6.2 & 1.4 & 2.2 & 4.4 & 8.1 & 6.7 & 8.3 & 9.4 & 2.8 \\
\hline
\end{array}
\end{aligned}
$$

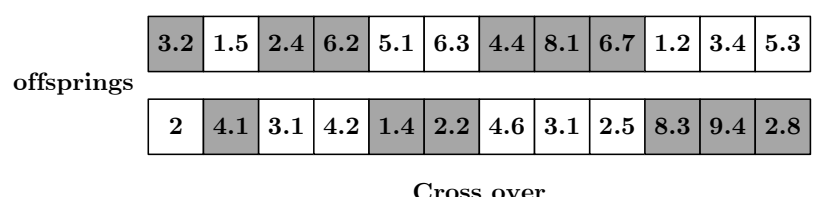

$$
\begin{aligned}
& \begin{array}{|c|c|c|c|c|c|c|c|c|c|c|c|}
\text { Parent } 2 & 1.5 & 3.1 & 4.2 & 5.1 & 6.3 & 4.6 & 3.1 & 2.5 & 1.2 & 3.4 & 5.3 \\
\hline
\end{array} \\
& \begin{array}{l|l|l|l|l|l|l|l|l|l|l|l|l|}
\text { offspring } 2 & 0.5 & 0.8 & 4.2 & 1 & 6.3 & 4.6 & 0.1 & 0.7 & 0.9 & 3.4 & 5.3 \\
\hline
\end{array} \\
& \text { Mutation }
\end{aligned}
$$

Figure 5 Real-coded genetic algorithm operators 
Scattered crossover operator is used for minimizing overturning moment. Just like nature, superior populations have more election possibility and effects on their perimeter. To model the process of evolution in the algorithm, fitness function is defined as a combination of overturning moment and fluid c.g. height. Using this application, the population with greater fitness, has more effect on the future. In generation $t$, evolutionary algorithm consists of $p$ chromosomes in the population. In $t=1$, the number of $N$ initial population is randomly generated. The produced target populations are decoded, and for each of them correspondingly a fitness function is considered. In order to solve optimization problems different methods such as real-coded and bit string is utilized. The GA real-coded technique is applied in the present optimization problem. Therefore, the calculations are based on real value of variables. The Figure 6 represents a diagram that investigate elliptical cross-section that give more stability to tank vehicle. Some constraints such as cross-sectional area and bounds of variables lead into the minimization. The variables of $a$ and $b$ in this diagram equal to the width and height values. This optimization determines two variables as fluid c.g. height $\left(Y_{c g}\right)$ and overturning moment $\left(M_{0}\right)$ in order to calculate the objective function as:

$$
U(X)=\text { Minimize }\left[w_{1} M_{0}+w_{2} Y_{C G}\right]
$$

The algorithm specifications such as theobjective function, constraints, design variables and their lower and upperbounds are briefly mentioned in Table 2 . The present method leads to selecting optimum variables and curving shapes that minimize overturning moment and fluid c.g. height.

Table 2 The Specifications of the genetic algorithm

\begin{tabular}{cc}
\hline \multicolumn{2}{c}{ The genetic algorithm characteristics } \\
\hline objective function & $U=w_{1} M_{0}+w_{2} Y_{c g}$ \\
tank variables & $\operatorname{tank}$ width $(2 b)$ \\
& $\operatorname{tank}$ height $(2 a)$ \\
optimization non linear constraint $\left(\mathrm{m}^{2}\right)$ & Area $(A=\pi a b \leq 6.4)$ \\
optimization linear constraint $(\mathrm{m})$ & $0.8 \leq a \leq 1.8$ \\
& $1.1 \leq b \leq 2.1$ \\
\hline
\end{tabular}

Latin American Journal of Solids and Structures 11(2014) 113 - 129 


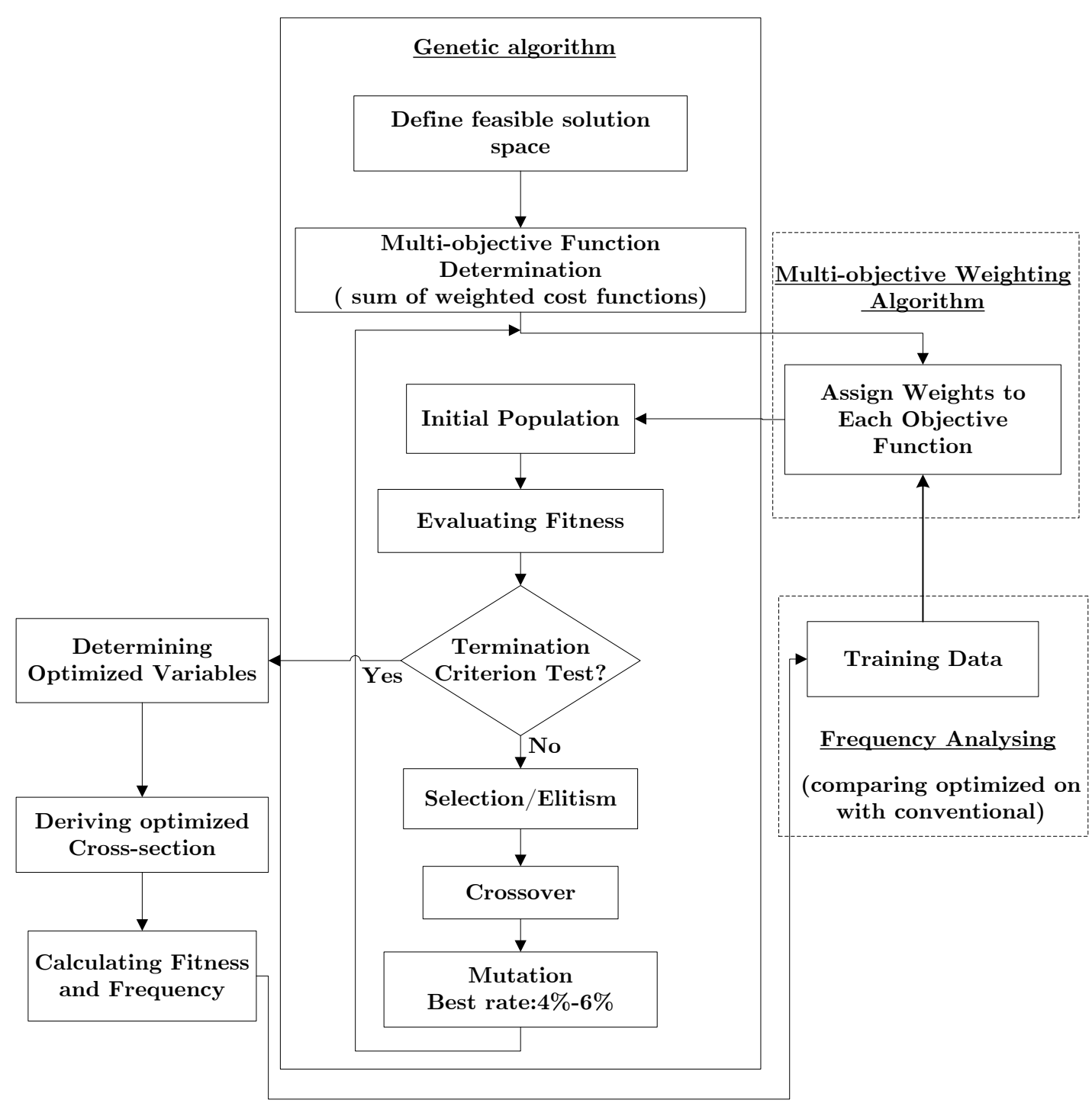

Figure 6 Flowchart of optimization using GA

The present multi-objective optimization method is converted to a single objective function based on sum of weighted cost function method. The weighting coefficients are chosen according to their importance (Kaur and Bakhshi, 2010; Haupt, 2004). The approach of Assign Weight To Each Objectives is used to determine different values assumptions for each sub-population on the relative worth of cost functions. The two vector sizes are determined in the case of a bi-criteria research (Pei-Chann and Chen, 2009). The weighted vector and cost functions are presented as follows:

$$
F=w_{m 1} f_{1}+w_{m 2} f_{2}, \quad f_{1}=Y_{c g}, f_{2}=M_{0}
$$




$$
\left(w_{m 1}, w_{m 2}\right)=\left(\frac{1}{m}, 1-\frac{1}{m}\right), \quad m=2,3 \ldots
$$

where $f_{1}$ and $f_{2}$ are the objective functions in each generation and $m$ is the $m$ th stage of choosing weighting coefficients. The Eq. (26) is obtained from studying the effects of objective functions on roll stability, in this paper. This process is repeated until the correct answers have been achieved. The obtained frequency and overturning moment are compared with the data of conventional elliptical tank, in order to omit unsuitable weighting coefficients through the algorithm which is shown in Figure 6.

\subsection{Developing Genetic Algorithm}

In order to find the best overturning moment that enhances roll stability of tankers a MATLAB code matching genetic algorithm is developed. Then, tank sloshing frequency is analyzed to avoid resonance phenomenon. In order to find the best elliptical tank shape with the highest rollover threshold, both elliptic parameters indicated as $a$ and $b$ (elliptic width and height) are introduced as unknown parameters, where the corresponding area is constrained. Moreover, the code has ability to investigate different design variables such as tank cross-sectional area, oval diameter, overturning moment, fluid c.g. height and sloshing frequency. The flowchart of genetic algorithm demonstrated in Figure 6, can be applied to achieve and accurate reliable results with genetic operators (mutation, cross over and etc). Individuals generated randomly in each generation, indicate overturning moment and fluid c.g. height simultaneously. They have the possibility of being out of feasible tank dimension. Therefore, to reduce the iteration number of generations and prevent improper individuals in population, a conventional tank shape and also some constraints are introduced for elliptical shape. Moreover, There are many points that have a low overturning moment in domain but they are not appropriate to be as an elliptic tank shape. These points lead into increasing the fluid c.g. height. In this respect, the objective function is determined as a combination of fluid c.g. height and overturning moment.

\subsection{Analyzing the Mutation Rate}

In this section, the mutation rate, which determines accuracy and speed of convergence response in the GA program, is investigated. The code developed here, includes a large number of explorations for different items such as population number, iteration number, value of cost function, and mutation rate. Thus, minimum value of iteration and speed of convergence are very important. To explore the effect of mutation rate an elliptical tank shape with the area of $3.256\left(\mathrm{~m}^{2}\right)$ is taken. The mutation rates are taken the values of $0,2 \%, 4 \%, 6 \%$, and $10 \%$. As presented in Figure 7 , without any mutation rate, an early convergence occurs and GA algorithm cannot satisfy all the possible value of constraints. For the case of $2 \%$ mutation the individual bit changes and the chance of achieving better fitness functions increases. While, the mutation equals zero, the best fitness function is obtained at 53 generation and the minimum overturning moment will be 55000 N.m. However, while the value of mutation rate takes $2 \%$, proper fitness function is obtained at 81 generation and the minimum overturning moment is 560000 N.m. As represented in Figure 7 ,

Latin American Journal of Solids and Structures 11(2014) 113 - 129 
enhancement of the mutation rate value increases the probability of various individuals in the population. Based on this explanation, the mutation rate should be selected between $4 \%$ and $6 \%$. Moreover an appropriate population number would affect on achieving the best overturning moment. To explore the effect of population number and mutation rate, two filling levels of $70 \%$ and $80 \%$ of an elliptical container are studied.

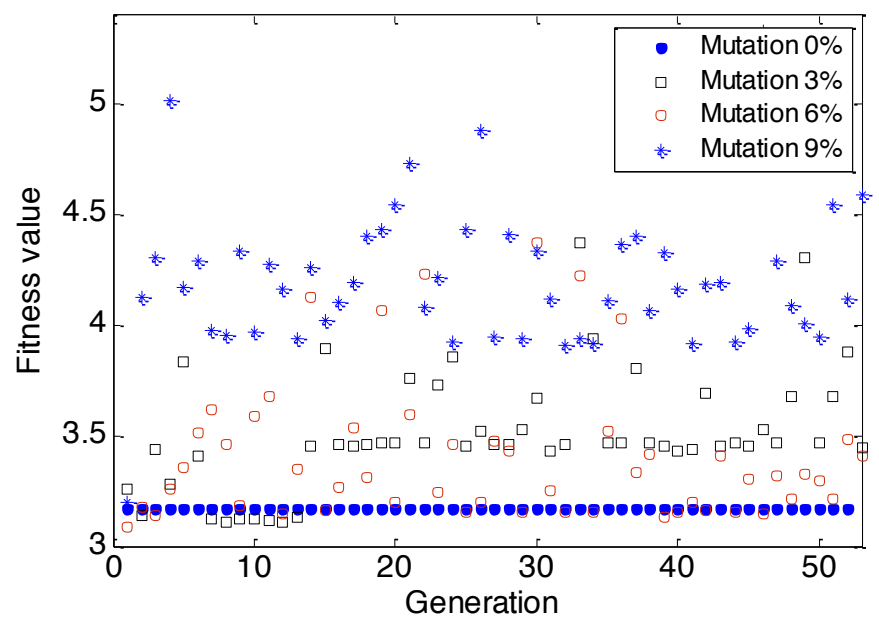

Figure 7 Comparing convergence of fitness function in different mutation

As illustrated in Figure 8, for all mutation rates except $0 \%$ and $10 \%$, the results are well converged. However, while the population is fewer than 40 or mutation rate is fewer than $2 \%$ and over $10 \%$, fitness functions that represent overturning moment and as a result frequency is not compatible. Therefore, the population number should be at least 40 .

The results of presented research based on real-coded GA are compared with other tank vehicles. As shown in Figure 9overturning moment of differrent tank shapes such as circular tank and conventional elliptical tank are compared with optimized elliptical tank shape. Results show that optimized tank shape has minimum overturning moment compared to other tank shapes. In other hand, using optimized GA real-value method increase tank stability, although it has a reverse effect on sloshing frequency.

The natural sloshing frequencies of optimized elliptical tank are compared with those of conventional elliptical and circular ones that are obtained based on numerical and analytical analyzes (Figure 10). It is well observed that converting the cylindical shapes into elliptical one decreases the natural frequency of the sloshing mode which is not suitable. It also depicts that the natural frequency of optimized elliptical shape is somewhat higher than conventional ones.

In this study, two different filling conditions ( $70 \%$ and $80 \%)$ of optimal elliptical tank that is obtained based on conventional circular forms MC307 and MC312 with a diameter of 2.03 mare illustrated. After analyzing related optimizing results, it is concluded that optimal elliptical crosssection for both filling conditions has the best convergence in 120 individual numbers. The optimal cross-section is presented in Figure 11 As depicted in this figure the oval parameters $a$ and $b$ obtained for instance in $90 \%$ filling level, the optimal oval diameters are equal to $a=1.6$ and 
$b=1.28 \mathrm{~m}$, which they are optimized values of a circular with radius of $1.427 \mathrm{~m}$. The convergence is obtained after 102 iterations.

70 Percent Filling Level
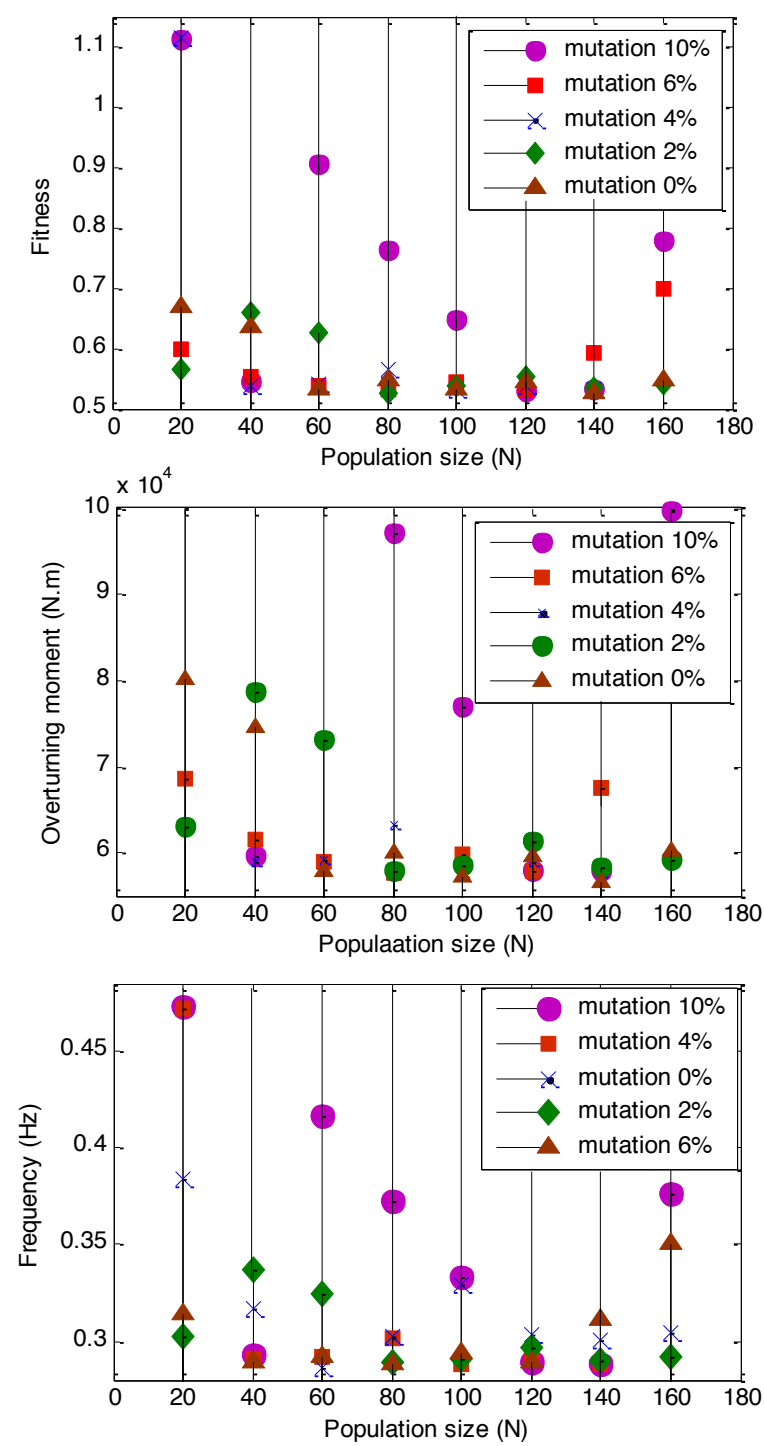

80 Percent Filling Level
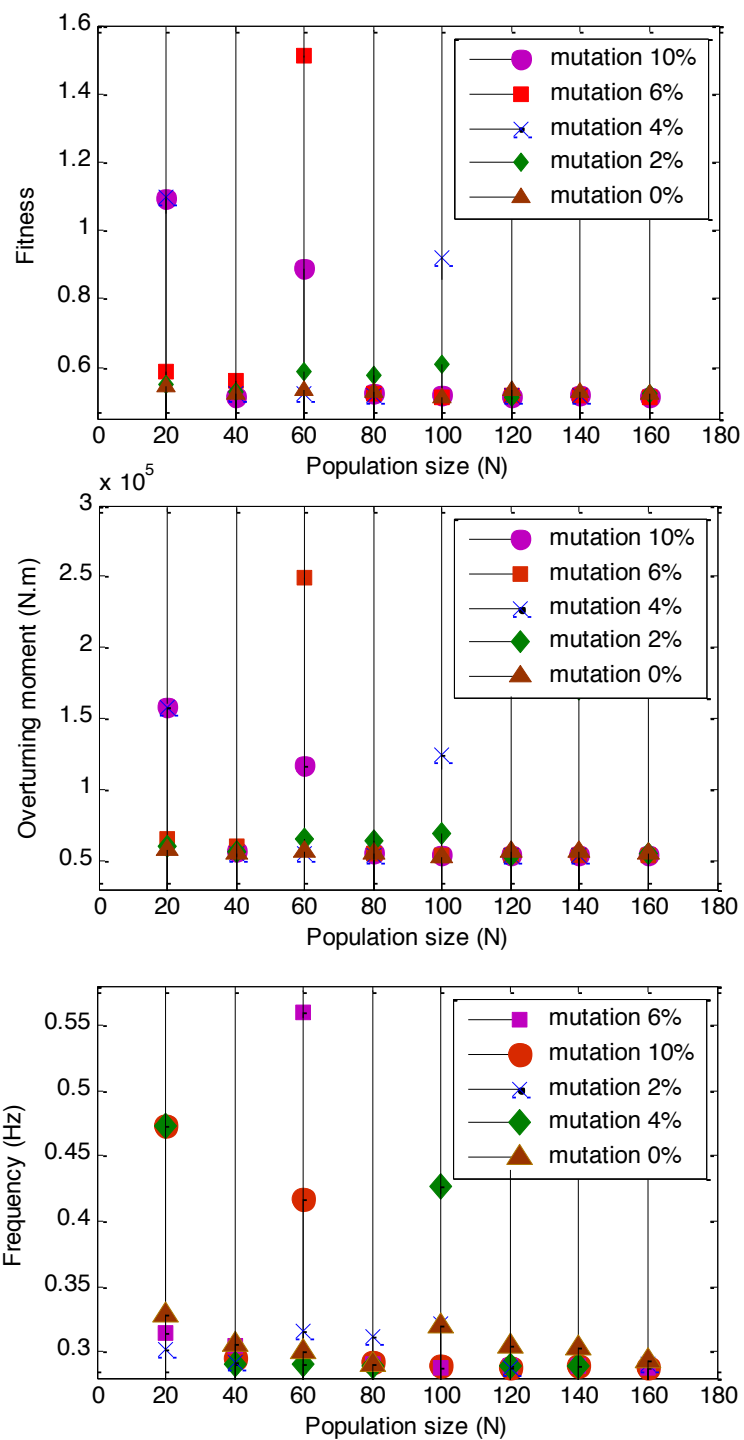

Figure 8 Fitness function, overturning moment and frequency in different population size and mutation rate 


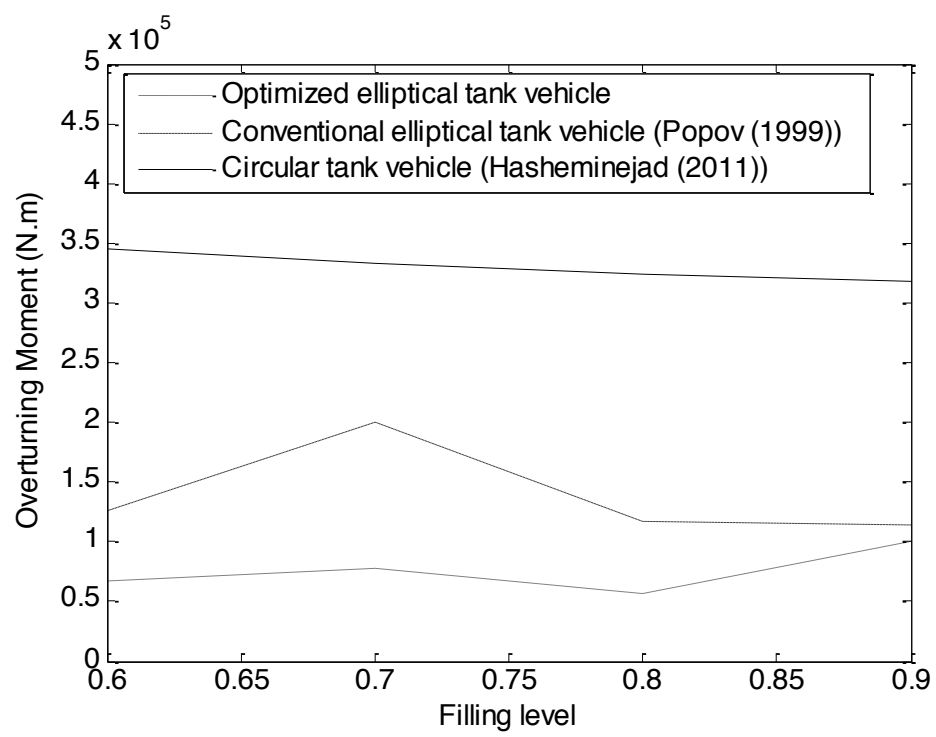

Figure 9 optimal elliptical overturning moment

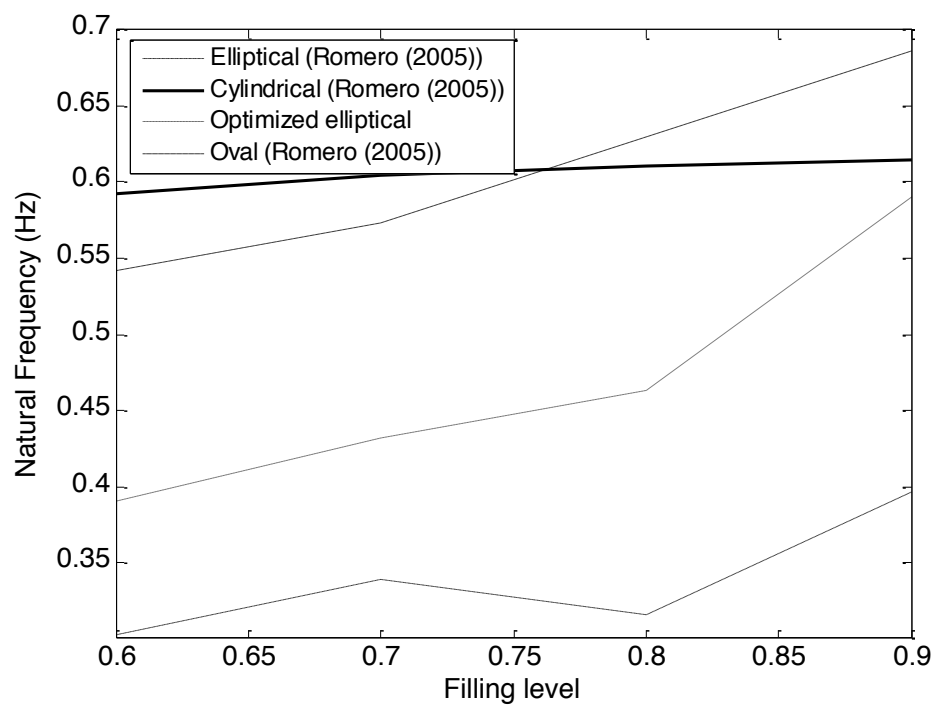

Figure 10 Natural frequency in different shape 


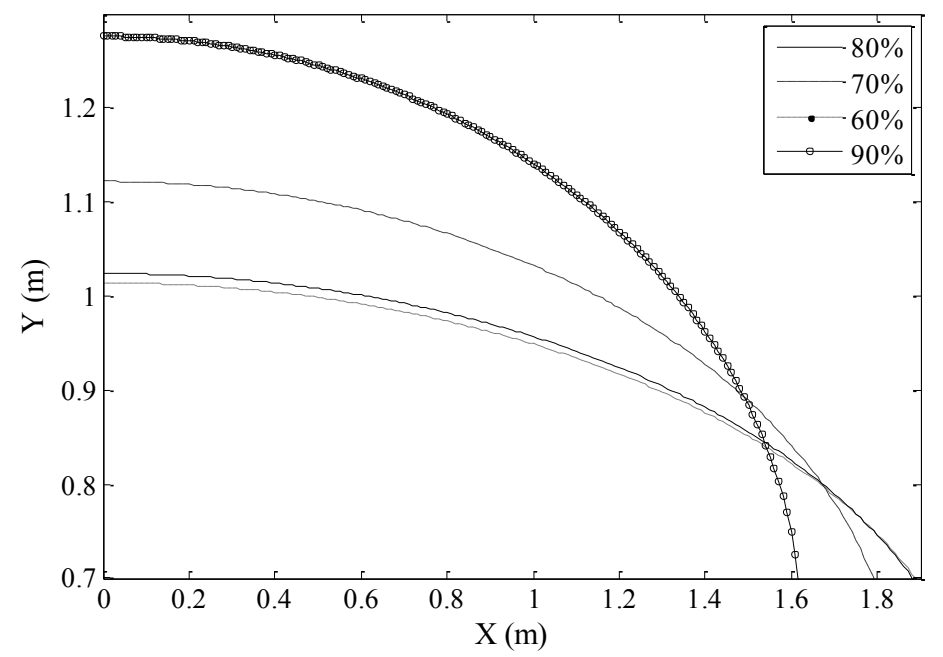

Figure 11 Optimized elliptical corss-sections in different filling conditions

\section{CONCLUSION}

In this paper, a method based on real-coded genetic algorithm was proposed to specify the best elliptical cross-section with minimum fluid c.g. height and overturning moment in the feasible tank cross-sectional area. In this reason, a code was developed to predict the optimized elliptical cross-section. In addition natural sloshing frequency was considered as a factor of tank rollover. The results presented in different filling conditions were compared with conventional methods. Processing the results indicates that the present method is a suitable way to determine optimized elliptical tank shape. The results also show that the natural sloshing frequency is enhanced in comparison to conventional elliptical tanks. It is a worthwhile result, as it indicates the roll stability of optimized tank in a stable margin.

The results also indicated that the population number should be at least 40 and mutation rate is suggested $4 \%$ to $6 \%$. Finally, it should be noted that the proposed model is a fast and accurate method to design elliptical tank shape, which increases natural sloshing frequency in compare with conventional elliptical tank cross-sections. 


\section{References}

Acarman, Tankut, (2006). Rollover prevention for heavy trucks using frequency shaped sliding mode control. Vehicle System Dynamics. 44:737-762.

Aliabadi, S., Johnson, A., Abedi, J., (2003). Comparison of finite element and pendulum models for simulation of sloshing. Computer and Fluids. 32:535-545.

Budiansky, B., (1960). Sloshing of Liquids in Circular Canals and Spherical Tanks. Journal of the Aerospace Sciences. 27:161-173.

Hasheminejad, M., Aghabeig, M., (2011).Transient sloshing in half-full horizontal elliptical tanks under lateral excitation. Journal of Sound and Vibration. 330:3507-3525.

Hyun-Soo Kim, (2008). Optimization design technique for reduction of sloshing by evolutionary methods. Journal of Mechanical Science and Technology. 22:25-33.

Ibrahim, R. A., Barr, A. D. S., (1975). Autoparametric resonance in a structure containing a liquid, partI: two mode interaction. Journal of Sound and Vibration. 42:159-179.

Ibrahim, R. A., Li, W., (1988). Parametric and autoparametric vibration of an elevated water tower, part II: Autoparametric response. Journal of Sound and Vibration. 121:429-444.

Iekda, T., Nalagawa, N., (1997). Nonlinear vibration of a structure caused by water sloshing ina rectangular tank. Journal of Sound and Vibration. 201:23-41.

Kang, X., Rakheja, S., Stiharu, I., (1999). Optimal tank geometry to enhance static roll stability of partially filled tank vehicles. SAE paper No. 1999-01-3730:542-553.

Kaur ,A., Bakhshi, A. K., (2010). Change in optimum genetic algorithm solution with changing band discontinuities and band widths of electrically conducting copolymers. Chem Phys. 369:122-125.

Li, Yuchun, Wang, Jinting, (2012). A supplementary, exact solution of an equivalent mechanical model for a sloshing fluid in a rectangular tank. Journal of Fluids and Structures. 31:147-151.

Pei-Chann, Chang, Shih-Hsin, Chen, (2009), The development of a sub-population genetic algorithm II (SPGA II) for multi-objective combinatorial problems. Applied Soft Computing. 9:173-181.

Popov, G., Sankar, S., Sankar, T. S., (1996). Shape optimization of elliptical road containers due to liquid load in steady-state turning. Vehicle System Dynamics. 25:203-221.

Rakheja, R., Sanker, S., Ranganthan, R., (1988). Roll plane analysis of articulated tank vehicles during steady turning. Vehicle System Dynamics. 17:81-104.

Rakheja, S., Stiharu, I., Kang, X., Romero, J.A., (2002). Influence of tank cross-section and road adhesion on dynamic response of partly filled tank trucks under braking-in-a-turn. Journal of Vehicle Design. 9:223-240.

Haupt, Randy L., Haupt, Sue Ellen, (2004). Practical genetic algorithms, Second ed. John Wiley \& Sons, New Jersey.

Romero, J., Hildebrand, A. R., Martínez, M., Ramírez, O., Fortanell, J. M., (2005).Natural sloshing frequencies of liquid cargo in road tankers.Int. J. of Heavy Vehicle Systems.12:121-138.

Romero, J. A., Lozano, A., Ortiz, W., (2007). Modelling of liquid cargo-vehicle interaction during turning manoeuvres. 12th IFToMM World Congress. Besançon (France).

Salehi, Mojtaba, Tavakkoli-Moghaddam, Reza, (2009). Application of genetic algorithm to computer-aided process planning in preliminary and detailed planning. Engineering Application of Artificial Intelligence. 22:1179-1187.

Sette, S., Boullart, L., (2001). Genetic programming: principles and applications. Engineering Application of Artificial Intelligence. 14:727-736. 